\title{
Registration of Point Cloud Data Based on PSO
}

\author{
Xianquan $\operatorname{Han}^{1,2, a}$ \\ ${ }^{1}$ Yangtze River Scientific Research Institute, \\ Wuhan, 430010, China \\ ${ }^{2}$ Key Laboratory of Precise Engineering and Industry \\ Surveying of National Administration of Surveying, \\ Mapping and Geoinformation, \\ Wuhan, 430079, China; \\ amydabao112@hotmail.com
}

\author{
Zan $\mathrm{Nie}^{3, \mathrm{~b}}$ \\ ${ }^{3}$ Power China Hubei Electric \\ Engineering Corporation, \\ Wuhan 430040, China \\ bniez@heec.com
}

\author{
Zhen Zhang ${ }^{4, c}$ \\ ${ }^{4}$ Xinjiang Investigation and Design \\ Institute of Water Conservancy and \\ hydropwer, \\ Wulumuqi, 830000, China \\ czhangzhen_soul9@sina.com
}

\author{
Li Zhou ${ }^{5, d}$ \\ ${ }^{5}$ Hubei province Zhanghe Project \\ Management Office, \\ Jingmen, 448000, China \\ d371914623@qq.com
}

\begin{abstract}
At present,3-D model can be build through point clouds data which is quickly get by laser scanning technology. Most of the researches on registration problems are focused on feature matching method. This paper discusses a method with global optimization algorithm. In order to solve the problem efficiently, we present a method using PSO algorithm with Cross Products of Normal Vector. This method does not require artificial targets and feature points frequently. The fitness function is used the sum of normal vectors' cross products to define, and it applies an efficient "Universal Search" and implements cloud data registration based on the best registration criterion. The application and analysis on example indicates the proposed method is effective and feasible.
\end{abstract}

Keywords-point cloud; registration; PSO; cross products of normal vector.

\section{INTRODUCTION}

3-D laser scanning is a technology revolution in surveying and mapping field after the GPS. This technology is able to get the point data with high precision and density, so it is widely used in reverse engineering, surveying and mapping engineering, ancient architecture protection, and 3-D city modeling in recent years[1].If 3-D laser technology is applied to engineering practice in production, it can improve the work efficiency and can be used as core technique in automated logging to provide a new way for fast accurate and automated surveying and mapping[2].

Measure in using 3-D laser scanner, a complete object data acquisition needs more station scans, so the registration technology of the many scans is the key technology of data processing. The traditional registration method usually extract feature points in point cloud, using quaternion for the calculation of transformation parameters[3].But the point cloud data is discrete, feature points is not obvious, so it is difficult to extract characteristics point data automatically.The registration results can not meet the need of 3-D modeling sometimes, since the feature and characteristics of point data are not obvious. This paper advances a kind of fast registration method, it puts forward the object of the point cloud data as a whole and use the normal vector information of point cloud based on PSO algorithm. The search and registration is going on in the global scope in order to get a better matching effect.

The paper is organized as follows. In the next section, we propose the problems and technology in the point cloud registration. In Section 3, the registration criterion is presented. Section 4 presents the PSO algorithm steps. In Section 5, the computational example and analysis is made to illustrate the efficiency of the method. Finally, we conclude our paper in section 6 .

\section{DISCUSSED PROBLEMS}

Point cloud registration is a key technology in 3-D modeling[4,5].In the present study point cloud registration algorithms are based on "feature matching" as well as the ICP and its improved algorithms. According to the coordinates of corresponding feature points, the transformation parameters of the two views can be calculated[6,7].For the point cloud data, feature extraction algorithm is not mature in itself, it is unable to accurately extract all feature in huge point cloud data all the time, thus the calculation of transformation parameters will also have the deviation. After the splicing multi-stations, the error will be accumulated, it is adverse for modeling $[8,9]$. 
Because of point cloud data is huge, it is difficult to match the all the data dir-ectly, even the point cloud and their corresponding ground point in actual may not have the same feature point. If the selection of feature point is inaccurate, it may bring great errors to the results. The problem in point cloud registration is the study need to solve.

\section{REGISTRATION CRITERION}

In the process of obtaining point cloud data, not only has the coordinates of point cloud data, and the normal vector information of them. Relative to the coordinate information, the normal vector information is better to reflect the spatial character-ristics of the point clouds. In the complete registration situation, the normal vect-or at different stations in theory should be paralleled to each other. By the theo-ry of analytic geometry, parallel to the normal vector of the cross pro-duct is 0 .

The formula is written as Eq. 1 shows:

$$
\vec{a} \times \vec{b}=\overrightarrow{0}
$$

$$
\left[y_{1} z_{2}-z_{1} y_{2}, z_{1} x_{2}-x_{1} z_{2}, x_{1} y_{2}-y_{1} x_{2}\right]=[0,0,0]
$$

Where $\vec{a}\left(x_{1}, y_{1}, z_{1}\right) 、 \vec{b}\left(x_{2}, y_{2}, z_{2}\right)$ are the normal vectors .

In the registration, if the two groups of point cloud data are matched very well, all its point cloud normal vectors should be paralleled to each other. For the different station point cloud data, the algebraic sum of absolute value about vector cross product should be 0 .But due to the instrument and environmental factors effect, there is some error actually, the algebraic sum of absolute value about vector cross product can only be relatively minimal. The formula is written as Eq. 2 shows:

$$
f\left(P_{i}\right)=\min \sum\left(\left|y_{i 1} z_{i 2}-z_{i 1} y_{i 2}\right|+\left|z_{i 1} x_{i 2}-x_{i 1} z_{i 2}\right|+\left|x_{i 1} y_{i 2}-y_{i 1} x_{i 2}\right|\right)
$$

Where $P_{i}$ is a random position in point cloud data.

The registration process use all the vector information of the point cloud data, not only the individual feature in the overlap region are calculated. So the registr-ation results can achieve optimal overall. Compared with the conventional regist-ration methods, the calculation method of the cross product of vectors is relativel-y simple, fast and high precision.

\section{PSO ALGORITHM}

Particle swarm optimization(PSO) is a new evolutionary algorithm developed in recent years[10].It is initialized to a group of random particle, and then through the iteration to find the optimal solution (fitness), in each iteration, the particle is updated by two "values". First is the optimal particle itself which is called pbest, another is the whole population found in the optimal solution, the global

value which is called gbest.

The formula is written as Eq.3 shows:

$$
\left\{\begin{array}{c}
v_{i}(t+1)=v_{i}(t)+c_{1} r_{1}(t)\left(\text { pbest }_{i}-x_{i}(t)\right)+c_{2} r_{2 j}(t)\left(\text { gbest }-x_{i}(t)\right) \\
x_{i}(t+1)=x_{i}(t)+v_{i}(t+1)
\end{array}\right.
$$

Where $c_{1} 、 c_{2}$ are the non-negative learning parameters, $r_{1}, r_{2}$ are random numbers in $(0,1)$,used to keep the diversity.

Set $X_{i}=\left(x_{i 1}, x_{i 2}, \cdots, x_{i n}\right)$ is the current location of particle $\mathrm{i}, V_{i}=\left(v_{i 1}, v_{i 2}, \cdots, v_{i n}\right)$ is the current velocity of particle i, $P_{i}=\left(p_{i 1}, p_{i 2}, \cdots, p_{i n}\right)$ is the best position of the particle $v_{i}$ which is also the particles experienced with the best fitness value position so far, called pbest. For the minimization problem as well as Eq. (2), if the value of the objective function is the smallest, the corresponding fitness value we can get is better.

Set $f(X)$ to minimize the objective function.Hence, Eq.(2) can be transformed into Eq.(4):

$$
P_{i}(t+1)=\left\{\begin{array}{c}
P_{i}(t), f\left(P_{i}(t+1)\right) \geq f\left(P_{i}(t)\right) \\
P_{i}(t+1), f\left(P_{i}(t+1)\right)<f\left(P_{i}(t)\right)
\end{array}\right.
$$

Set the number of particles in the group is $s$,the best position of particle groups is $P_{g}(t)$,just like the global best position "gbest". The formula is written as Eq.5 shows:

$P_{g}(t) \in\left\{P_{0}(t), P_{1}(t), \cdots ; P_{s}(t) \mid f\left(P_{g}(t)\right)=\min f\left(P_{0}(t), P_{1}(t), \cdots ; P_{s}(t)\right)\right)$

Hence, Eq.(3) can be transformed into Eq.(6):

$$
\left\{\begin{array}{c}
v_{i j}(t+1)=v_{i j}(t)+c_{1} r_{1 j}(t)\left(P_{i j}(t)-x_{i j}(t)\right)+c_{2} r_{2 j}(t)\left(P_{g i}(t)-x_{i j}(t)\right) \\
x_{i j}(t+1)=x_{i j}(t)+v_{i j}(t+1)
\end{array}\right.
$$

Where the $P_{i j}(t)$ is a local best position of the particle as well as pbest. $P_{g i}(t)$ is the optimal position of the whole particle swarm so far to search as well as gbest.By updating the particle's position,it can find the best position through the calculation method of the cross product of vectors.

The algorithm process is as follows:

Step1: Set the particle swarm random position and velocity initially.

Step2: Calculate the each particle fitness value .

Step3: Compare each particle's fitness with the $P_{i j}(t)$.If better than before, update the best position in the current.

Step4: Compare each particle's fitness with the $P_{g i}(t)$.If better than before, update the global position in the current.

Step5: Update the position and velocity of a particle.

Step6: Judge the termination condition of algorithm is satisfied or not, then output the global optimal solution of $P_{g i}(t)$,otherwise turn to Step3 continue to iterate.

Step7: Using $P_{g i}(t)$,namely the optimal transformation parameters for the calculation of point cloud data, get the final registration of point cloud.

\section{COMPUTATIONAL EXAMPLES AND ANALYSIS}

In order to illustrate the method's effectiveness, we construct a scanning of a quarry using the MATLAB toolbox function to solve the registration problem. After the elimination of noise data, it made a rough registration, and then used the PSO algorithm with criterion for accurate registration and adjustment. 
Through two-registration, there are some main views with larger overlap area will be registered firstly, which can be registered with adjacent overlap the largest view secondly. Whole registration requires sequence splicing stations in order to satisfy the conditions of closed traverse, at the same time, the amount of translation and rotation Angle in the transformation parameters should be independent. The registration results will be verified through the closed error of closed traverse.

The results of the registration base on PSO are shown as follows:

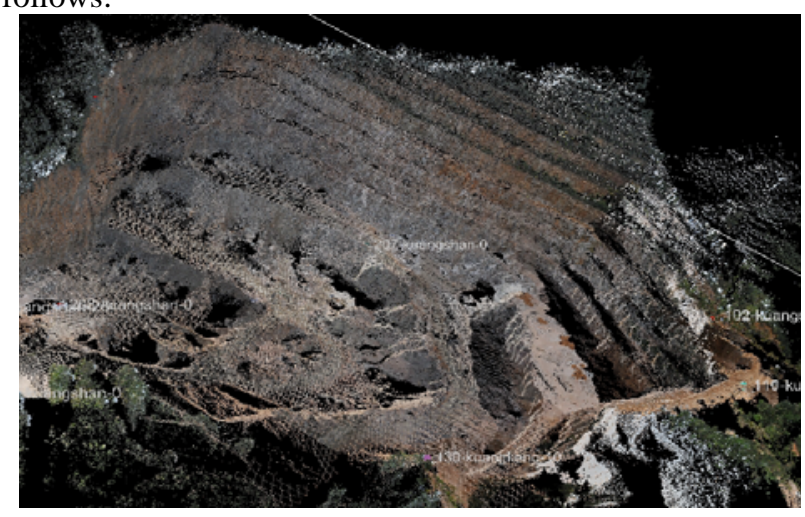

Figure 1. Point Clouds After Registration

TABLE I. THE CLOSED ERROR OF THE REGISTRATION

\begin{tabular}{|c|c|c|}
\hline $\begin{array}{c}\text { Closed error of } \\
\text { traverse }\end{array}$ & feature matching & PSO algorithm \\
\hline $\mathrm{X}$ & -0.0296 & 0.0082 \\
\hline $\mathrm{Y}$ & 0.0021 & -0.0013 \\
\hline $\mathrm{Z}$ & -0.0376 & -0.0024 \\
\hline$\alpha$ & -0.028 & -0.0053 \\
\hline$\beta$ & 0.042 & 0.0064 \\
\hline$\gamma$ & -0.002 & -0.0013 \\
\hline
\end{tabular}

As can be seen from Figure 1 and Table I, the optimal results shows that the PSO algorithm is feasible and efficient. Because of the feature extraction steps are omitted, the closed error of PSO algorithm is smaller, the reliability and accuracy of registration have been greatly improved.

\section{CONCLUSIONS}

Through theoretical analysis and experiment, the registration method based on PSO is proposed in this paper, which does not need the feature points in the calculation, as long as the adjacent station data should have some degree of overlap, so it can achieve high precision. The major advantage of this method is that it can effectively solve the problem like features are not easy to find. Hence, it can be used in condition where artificial targets and feature points is invisible or non-existent.

\section{ACKNOWLEDGEMENTS}

The research work was supported by National Natural Science Foundation of China under Grant No.41301434 and Open Foundation of Basic Scientific Research Operating Expenses of Central-Level Public Academies and Institutes (CKSF2013022/GC, CKSF2013020/GC,and Open Foundation of Key Laboratory of Precise Engineering and Industry Surveying of National Administration of Surveying, Mapping and Geoinformation (Grant No.PF2013-5)

\section{REFERENCES}

[1] Boulaassal H, Landes T, Grussenmeyer P, et al. Automatic segmentation of building facades using terrestrial laser data[J].International Archives of Photogrammetry, Remote Sensing and Spatial Information Systems,2007: 65-70.

[2] Lichti D, Stewart M P, Tsakiri M, et al. Calibration and testing of a terrestrial laser scanner[J].International archives of Photogrammetry and Remote sensing,2000, 33(B5/2; PART 5):485-492.

[3] Johnson A E, Bing Kang S. Registration and integration of textured 3D data[J]. Image and vision computing,1999, 17(2):135-147.

[4] Zheng Dehua, Shen Yunzhong, Liu Chun. 3D Laser Scanner and its effect factor analysis of Surveying error[J].Engineering of Surveying and Mapping,2005,14(2):32-34.

[5] Bergevin R, Soucy M, Gagnon H, et al. Towards a general multi-view registration technique[J].Pattern Analysis and Machine Intelligence,IEEE Transactions on, 1996, 18(5): 540-547.

[6] Xu Jinju, Zhang Yi, Wang Haicheng. Road surface surveying and data processing by 3D TLS[J].Bulletin of Surveying and Mapping,2011(11): 34-36.

[7] Park S Y, Subbarao M. An accurate and fast point-to-plane registration technique[J].Pattern Recognition Letters,2003,24(16): 2967-2976.

[8] Dorai C, Wang G, Jain A K, et al. Registration and integration of multiple object views for 3D model construction[J].Pattern Analysis and Machine Intelligence,IEEE Transactions on,1998,20(1): 83-89.

[9] Luo Xianbo, Zhong Yuexian,Li Renju. Data registration in 3-D scanning systems[J].Journal of Tsinghua University(Science and Technology),2004, 44(8): 1104-1106.

[10] Davis L. Handbook of genetic algorithms[J].1991. 